\title{
Optimal Tolerance Allocation for a Sliding Vane Compressor
}

\section{Yuan Mao Huang \\ Professor \\ e-mail: ymhuang@ntu.edu.tw}

\section{Ching-Shin Shiau \\ Research Assistant}

Department of Mechanical Engineering, National Taiwan University,

Taipei, Taiwan, Republic of China
An optimization model has been built with consideration of the required reliability, the minimum machining cost, and quality loss. The normal and the lognormal distributions of tolerances that depend on the production types of components are used in the reliability model. Cost tolerance data obtained from Bjфrke are used to calculate the machining cost. The asymmetric quadratic quality loss model is used to calculate quality loss caused by the deviation and the mean-shift of distributions. Tolerance allocation of a sliding vane rotary compressor is optimized for the required reliability, the minimum cost and quality loss, and optimum tolerances of components are recommended. The results show that high accuracies of the slot length, the vane thickness, and the slot width are required. Hence, their tolerances are smaller than other components. The effects of the correlation coefficient of the bottom cover plate and the top cover plate and the correlation coefficient of the front cover plate and the rear cover plate to total cost are insignificant. Further, the cost of quality loss is reduced when the weighting ratio of the quality loss function weighting coefficient to the machining cost function weighting coefficient is increased. The total cost is increased because tight tolerance allocation increases the machining cost. [DOI: 10.1115/1.2114893]

\section{Introduction}

Sliding vane rotary compressors have been developed and used for years because of lightweight, small size, simple mechanism, and easy maintenance. They are commonly used in refrigerators and automotive air conditioning systems. A considerable amount of studies were conducted to improve sliding vane rotary compressors [1].

A sliding vane rotary compressor has been designed and its schematic drawing is shown in Fig. 1 [2]. Some topics regarding this compressor were studied, such as the static contact force, stress and deflection of its vanes, stator contour, impact of vanes on the rotor, configuration, disassembly process, and test of the compressor [2-5]. The results showed that the compression ratio was not high as expected and the noise level was high because of leakage and friction due to improper tolerance.

Tolerance design is an important task in embodiment design. Tolerance allocation of an assembly in the conventional design is based on designers' experience or design handbooks. When tolerance is tight, the cost is high, and vice versa. If accumulated tolerance of components is out of specification and causes improper assembly, tolerances of components have to be revised. The task becomes complicated and very tedious if the number of components is large. Furthermore, the highest reliability, the best quality, and the minimum cost of a product cannot be assured and achieved.

The standard for the exchange of product model data (STEP) proposed by the international standard organization (ISO) and the product data exchange specification (PDES) proposed by the US National Bureau of Standards were very useful for tolerance design. Bjørke [6] and Juster et al. [7] studied the relationship of linear tolerance accumulation and allocation. Feng and Yang [8] generated a model to integrate tolerance information into the product database. Parkinson [9] applied a deterministic method of robust design to determine optimum nominal dimensions of components with dimensional tolerances. Zhou et al. [10] and Jordaan and Ungerer [11] used various numerical methods to approach optimal tolerance allocation. Di Stefano [12] analyzed statistical tolerance by using a mean shift model to evaluate the mean shift

Contributed by Design for Manufacturing Committee of ASME for publication in the Journal of Mechanical Design. Manuscript received May 1, 2004; final manuscript received May 1, 2005. Assoc. Editor: David Kazmer. factor. Hong and Chang [13] deal with interactions of the deviation space, deviation volume, and tolerance primitives to determine the relationship between process planning decisions and tolerances. Main and Ferreira [14] addressed the acceptable level of inaccuracy of the machined parts associated with its geometric tolerances. Manarvi and Juster [15] compiled available information and developed an integrated tolerance model and a tolerance allocation process. Hu et al. [16] provided automated methods for the specification of geometric tolerance types. $\mathrm{Wu}$ and Rao [17] investigated the modeling and analysis of tolerances and clearances. Mujezinovi'c et al. [18] applied a mathematical model for representing geometric tolerances to polygonal faces and showed its sensitivity to the precedence of datum reference frames.

Dong et al. [19] studied the relationship between tolerance and the machining cost. Shiu et al. [20] discussed misalignment and fabrication error of compliant parts and minimized manufacturing costs associated with tolerances. Xue and Ji [21] investigated a cost-tolerance model and allocated systematically the best tolerance to minimize the total manufacturing cost.

Mahadevan and Ni [22] analyzed tolerance reliability assessment of automotive spot-welded joints. Several researchers implemented the quality loss model [23-26] into tolerance synthesis. Ye and Salustri [27] constructed a nonlinear optimization model with consideration of product design, manufacturing, and quality simultaneously. Li [28] investigated the optimal manufacturing setting to minimize the expected quality loss.

Up to the present, only the combined cost tolerance and reliability model $[29,30]$ and the combined cost tolerance and the quality loss model $[23-25,31,32]$ were utilized for tolerance allocation. The optimal tolerance allocation model is not available based on the simultaneous consideration of the reliability, cost, and quality loss. In addition, semi-tolerances were assumed in existing studies. Therefore, the purpose of this study is to build an optimal tolerance allocation model with simultaneous consideration of the required reliability, the minimum cost, and quality loss. In the meantime, since tolerance allocation of the compressor as shown in Fig. 1 has not been optimized, tolerances between vanes and slots in the rotor, and vanes and the stator will be optimized. 


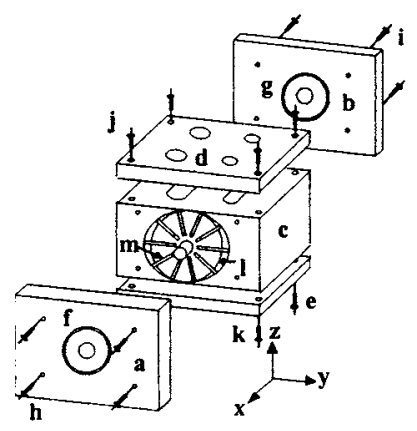

(a)

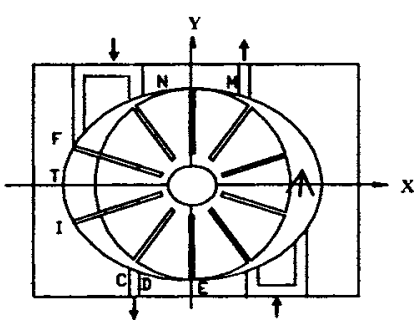

(b)
Fig. 1 Components and schematic drawing of sliding vane rotary compressor

\section{Method of Approach}

Basic components of the compressor as shown in Fig. 1 consist of a rotor, a stator, and sliding vanes. A rotor with ten slots for ten sliding vanes is inside and concentric with the stator. The outer surface of the rotor, the inner contour of the stator and the vanes form a volume segment. When the rotor rotates, the vanes slide outward along the slot due to the centrifugal force. The maximum volume segment is obtained at the end of the intake process. When the vanes slide inward, the volume segment is reduced and the air is compressed. The cross section of the compressor is shown in Fig. 2 and 18 independent components are 1 -stator, 2-rotor, 3-bottom cover plate, 4-top cover plate, 5-front cover plate, 6-rear cover plate, 7-vane, 8-front bearing, 9-rear bearing, 10-oil ring, 11-bottom bolt, 12-top bolt, 13-front bolt, 14-rear bolt, 15-bottom gasket, 16-top gasket, 17-front gasket, and 18-rear gasket.

The reliability index is the minimum distance from the origin of the coordinate system to the limited surface of the design. The standard parts of an assembly that are manufactured in mass production are considered as the normal distribution. The nonstandard parts of an assembly that are produced in batch production are considered as the lognormal distribution with a dimension mean-shift because of the asymmetric distribution [33].

Let $\bar{X}$ be the mean vector of the normal distribution vector or the lognormal distribution $X, V$ be the covariance matrix, and $\operatorname{cov}\left(x_{i}, x_{j}\right)$ stand for the covariance of the variable dimensions $x_{i}$ and $x_{j}$ in an assembly [34] as

$$
\begin{aligned}
& X=\left\{x_{1}, x_{2}, \ldots, x_{n}\right\} \\
& \bar{X}=\left\{\bar{x}_{1}, \bar{x}_{2}, \ldots, \bar{x}_{n}\right\}
\end{aligned}
$$

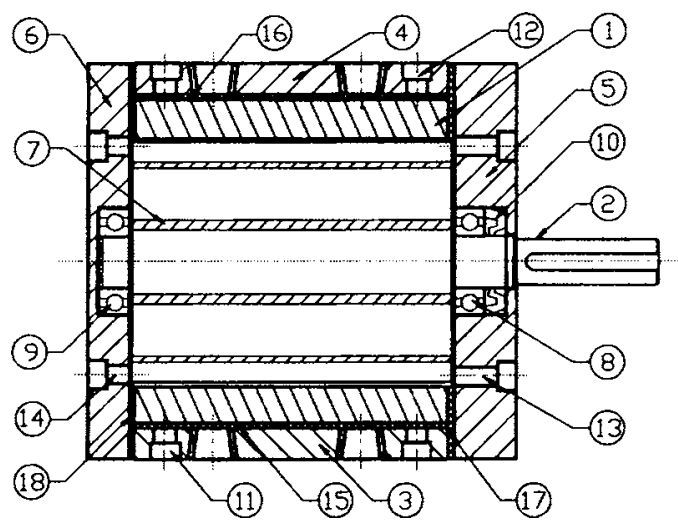

Fig. 2 Cross section of compressor and part numbers

$$
V=\left[\begin{array}{cccc}
\sigma_{1} & \operatorname{cov}\left(x_{1}, x_{2}\right) & \cdots & \operatorname{cov}\left(x_{1}, x_{n}\right) \\
\operatorname{cov}\left(x_{2}, x_{1}\right) & \sigma_{2} & \cdots & \operatorname{cov}\left(x_{2}, x_{n}\right) \\
\vdots & \vdots & \ddots & \vdots \\
\operatorname{cov}\left(x_{n}, x_{1}\right) & \operatorname{cov}\left(x_{n}, x_{2}\right) & \cdots & \sigma_{n}
\end{array}\right]
$$

where

$$
\operatorname{cov}\left(x_{i}, x_{j}\right)=E\left[\left(x_{i}-\mu_{i}\right)\left(x_{j}-\mu_{j}\right)\right]
$$

$\mu_{i}$ and $\mu_{j}$ are the mean values of $x_{i}$ and $x_{j}$, respectively, and $\sigma_{i}$ is the standard deviation of $x_{i}$. The coefficient of variation $\delta_{i}$ of $x_{i}$ is

$$
\delta_{i}=\frac{\sigma_{i}}{\mu_{i}}
$$

The variables of the normalized standard distribution for the normal distribution or the lognormal distribution, respectively, are

$$
y_{i}=\frac{x_{i}-\mu_{i}}{\sigma_{i}}
$$

and

$$
y_{i}=\frac{\ln x_{i}-\mu_{i}}{\sigma_{i}}
$$

The variable vector of the normalized standard distribution is $Y$ $=\left\{y_{1}, y_{2}, \ldots, y_{n}\right\}$. The correlation coefficient of $y_{i}$ and $y_{j}$ is

$$
\rho_{i j}=\frac{1}{N} \sum_{i=1}^{N} y_{i} y_{j}
$$

where $N$ is the population size. The independent standard normal distribution vector $Z=\left\{z_{1}, z_{2}, z_{3}, \ldots, z_{n}\right\}$ of variables $z_{i}$ can be obtained from

$$
\begin{gathered}
y_{1}=\alpha_{11} z_{1} \\
y_{2}=\alpha_{21} z_{1}+\alpha_{22} z_{2} \\
\cdots \\
y_{n}=\alpha_{n 1} z_{1}+\alpha_{n 2} z_{2}+\cdots+\alpha_{n n} z_{n}
\end{gathered}
$$

where

$$
\begin{aligned}
& \alpha_{11}=1.0 \\
& \alpha_{i 1}=\rho_{i j} \quad 1<i \\
& \alpha_{i k}=\frac{1}{\alpha_{k k}}\left(\rho_{i j}-\sum_{j=1}^{k-1} \alpha_{i j} \alpha_{k j}\right) \quad 1<k<i \\
& \alpha_{i i}=\sqrt{1-\sum_{j=1}^{i-1} \alpha_{i j}^{2}}
\end{aligned}
$$

Then the variables $z_{i}$ are

$$
\begin{gathered}
z_{1}=y_{1} \\
z_{2}=\frac{1}{\alpha_{22}}\left(y_{2}-\alpha_{21} z_{1}\right) \\
\ldots \\
z_{n}=\frac{1}{\alpha_{n n}}\left(y_{n}-\alpha_{n 1} z_{1} \ldots-\alpha_{n, n-1} z_{n-1}\right)
\end{gathered}
$$

Once the independent normal distribution vector $Z$ is available, the reliability index can be obtained by using the Beta algorithm which was developed by Hohenbichler and Rackwitz [35] as shown in Fig. 3, where $G$ is the design function. Details of the Beta algorithm are presented in the Appendix. 


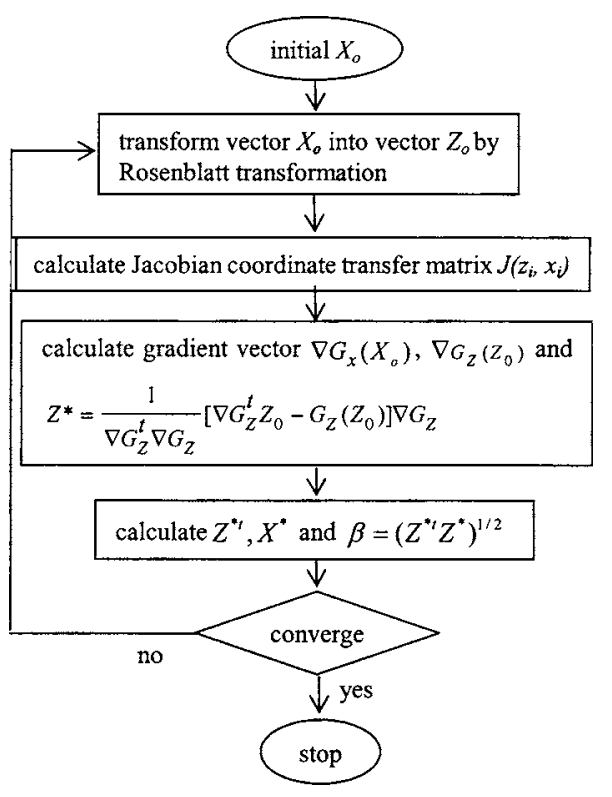

Fig. 3 Flowchart of beta algorithm process

In the case of multiple design functions, the minimum reliability index is

$$
\beta_{\min }=\min \left\{\beta_{1}, \beta_{2}, \ldots, \beta_{k}, \ldots, \beta_{p}\right\} \quad 1 \leqslant k \leqslant p
$$

where $p$ is the number of design functions.

The quality loss model of the-nominal-the-best type is that the best value of dimension is equal to the nominal dimension. If it is used, the expected total loss is [36]

$$
\begin{aligned}
E\left(L_{\mathrm{asm}}\right)= & \sum_{i=1}^{n} \\
& \times\left(\sum_{\mu_{i}<m_{i}} k_{i L}\left[\sigma_{i}^{2}+\left(\mu_{i}-m_{i}\right)^{2}\right]+\sum_{\mu_{i}>m_{i}} k_{i R}\left[\sigma_{i}^{2}+\left(\mu_{i}-m_{i}\right)^{2}\right]\right)
\end{aligned}
$$

where $m_{i}$ stands for the nominal value of the $i$ th dimension

$$
\begin{gathered}
k_{i L}=\frac{A_{h}}{\Delta_{i L}^{2}} \\
k_{i R}=\frac{A_{h}}{\Delta_{i R}^{2}}
\end{gathered}
$$

are quality loss coefficients, $A_{h}$ is the cost of the replacement or the repair for the $h$ th component, and $\Delta_{i L}$ and $\Delta_{i R}$ are specific semi-tolerance, or called customer's semi-tolerance of the $i$ th dimension. The quality loss curve and the probabilistic density function are shown in Fig. 4.

It can be seen that quality loss consists of loss by the deviation, $k_{i L} \sigma_{i}^{2}$ and $k_{i R} \sigma_{i}^{2}$, and loss by the mean-shift, $k_{i L}\left(\mu_{i}-m_{i}\right)^{2}$ and $k_{i R}\left(\mu_{i}-m_{i}\right)^{2}$. Quality loss increases when the standard deviation increases or the mean-shift increases.

Since the reliability index stands for the minimum distance from the origin of the coordinates to the limited surface in the standard normal distribution, the reliability corresponding to the reliability index can be obtained from the inverse of the accumulated distribution function based on the specified reliability. The left and right side semi-tolerances provide the required information related to quality loss. Söderberg [23] defined that the customer's lower acceptance limit of a component life is $75 \%$ of the nominal value. The reliability index of $75 \%$ reliability is

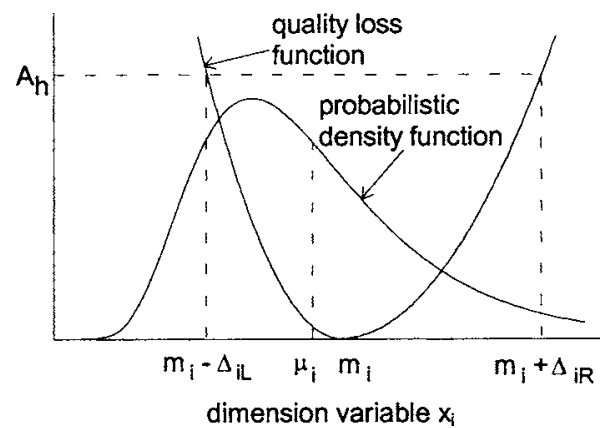

Fig. 4 Asymmetric quality loss curve and asymmetric dimension distribution

$$
\beta^{*}=\Phi^{-1}(0.75)=0.67449
$$

where $\Phi$ is the accumulated functional distribution of the independent normal distribution. Customer's semi-tolerance can be optimized for minimizing the goal of the machining cost through the machining cost-tolerance model $C_{m}(\Gamma)$ with the constraint

$$
\beta^{*}-\beta_{k}(\Gamma) \leqslant 0 \quad 1 \leqslant k \leqslant p
$$

where

$$
\Gamma=\left\{\sigma_{1}, \sigma_{2}, \ldots, \sigma_{i}, \ldots, \sigma_{n}\right\} \quad 1 \leqslant i \leqslant n
$$

The process of calculating the quality loss coefficient is shown in Fig. 5.

If the replacement cost $A_{h}$ is known, a set of $\Delta_{i L}$ and $\Delta_{i R}$ nearest to the specific customer's quality loss can be obtained from Fig. 4. In the meantime, the quality loss coefficients $k_{i L}$ and $k_{i R}$ of the asymmetric quality loss function can be obtained. Then tolerance can be optimized by a non-linear programming with minimizing the goal of

$$
C_{t}(\Gamma)=W_{m} C_{m}(\Gamma)+W_{q} E\left(L_{\text {asm }}(\Gamma)\right)
$$

and constraint function

$$
\beta^{* *}-\beta_{k}(\Gamma) \leqslant 0 \quad 1 \leqslant k \leqslant p
$$

where $C_{t}$ is the total cost that consists of the machining cost $C_{m}$ and the quality loss expected value $E\left(L_{\text {asm }}\right), W_{m}$ and $W_{q}$ are the weighting coefficients of the machining cost function and the quality loss function, respectively, $\beta^{* *}$ is the required assembly reliability index, and $p$ is the number of design functions. The reliability index $\beta_{k}$ of each design function stands for the assembly yield rate of each component. If $\beta^{* *}$ is larger, the reliability of the assembly will be higher. When the reliability is set at $99.0 \%$, the corresponding reliability index is 2.32635 . The processes to obtain semi-tolerance are shown in Fig. 6.

The minimization of machining cost and quality loss is a multiattribute decision-making problem. Also, the desired reliability in-

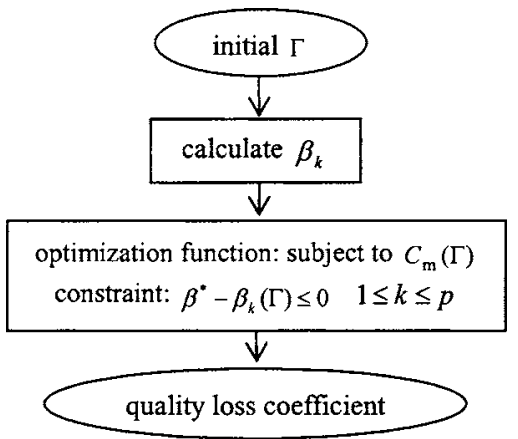

Fig. 5 Flowchart of calculating quality loss coefficient 


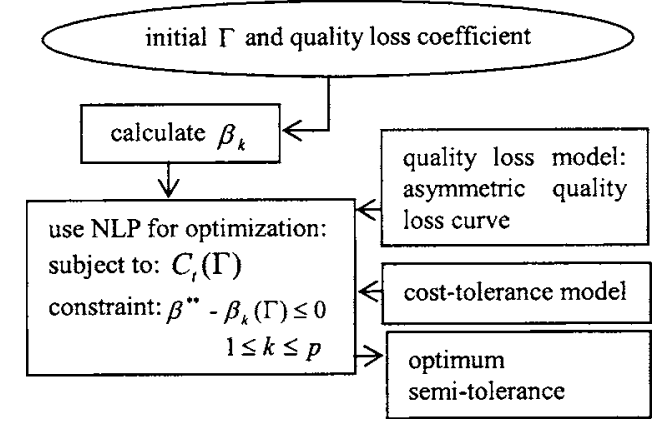

Fig. 6 Flowchart of determining optimal semi-tolerance

dex can be set in a penalty function form as the third objective function. Due to the consideration of computational efficiency, the attribute functions can be added together with different weighting coefficients. Cheng and Maghsoodloo [26] directly summed the cost-tolerance and quality loss for tolerance allocation, but they only considered the normal distribution condition without dimensional correlation. In this study, the replacement cost in the quality loss function has been carefully calculated in order to have an equivalent unit as machining cost in Bjørke's cost-tolerance model. Therefore, machining cost function and quality loss function can be combined into a single objective function with selected weighting coefficients. The weighting ratio $W_{q} / W_{m}$ represents the relative importance between quality loss and machining cost.

Oriented Functional Relationship. If an assembly that consists of many components is complicated, it is not worth analyzing all dimension features because of the cost. The assembly feature graph or the functional feature graph can be used to determine key functional dimensions. The representation of the oriented functional relation for dimension tolerance proposed by Zhang and Porchet [37] is used in this study, and its main purpose is to organize features of components according to assembly drawings. Users can find functional components, which really affect the performance of a product, quickly from the data bank and analyze tolerance features.

The oriented functional relationship is shown in Fig. 7. Each node stands for an independent component, and a line with an arrow stands for the mating relation between components. If an arrow points from $A$ to $B$, it stands for component $B$ is fixed on component $A$. A line without an arrow stands for a sub-assembly with components as points $C$ and $D$, and these components are assembled into a sub-assembly before being assembled with other components. A dashed line stands for the functional dimension that needs to be controlled for the dimension and tolerance, as point $E$ to point $F$.

Six symbols to represent relations between components are shown in Table 1. The connection between planes and the connection between cylinders stand for a tight fitting between two planes and two cylinders, respectively. The connection by planes and planes and the connection by cylinders and cylinders stand for a distance existed and loose running fit for planes and cylinders, respectively. The connection by cylinders and planes stands for simultaneous existence of connection between planes and connec-

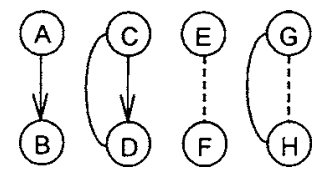

Fig. 7 Oriented functional relationship
Table 1 Symbols for feature fit in oriented functional relationship

\begin{tabular}{cc}
\hline Relation of connection & Symbol \\
\hline Connection between planes & $\mathrm{p}$ \\
Connection between cylinders & $\mathrm{c}$ \\
Connection by planes and planes & $\mathrm{pp}$ \\
Connection by cylinders and cylinders & $\mathrm{cc}$ \\
Connection by cylinders and planes & $\mathrm{cp}$ \\
Thread connection & $\mathrm{t}$ \\
\hline \hline
\end{tabular}

tion between cylinders.

The oriented functional relationship graph of the compressor as shown in Figs. 1 and 2 is shown in Fig. 8. The bottom cover plate that is used as the basis is at the top. Only a line contact, but without the assembly relationship, exists between vanes and the stator inner contour. The rotor connects to the inner rings of two bearings. Each bearing is considered as a sub-assembly that consists of the outer ring and the inner ring with the relative motion between them and cannot be counted as one node. Hence, nodes 8 and 9 are divided into $8_{1}$ and $8_{2}$, and $9_{1}$ and $9_{2}$, respectively. The rotor and vanes are considered as the third sub-assembly.

It is necessary to find tolerance chains of functional dimensions in order to analyze dimension tolerance. There are four dashed lines. If a functional dimension chain consists of these specific dimensions, all tolerances in the dimension chain should be analyzed. The dashed line from node 1 to node 7 stands for the contact between vanes and the stator inner contour. When the rotor is stationary, vanes may not contact with the stator. When the rotor rotates, vanes slide outward due to the centrifugal force. The tip of the vane slides along the stator inner contour. Although the dashed line represents the special contact situation in the oriented functional relationship graph, there actually is no functional tolerance between vanes and the stator. Therefore, it is not necessary to analyze this dimension chain.

Honda et al. [38] utilized a numerical method to analyze the gap flow between the side cover plate and the rotor and proved the importance of the clearance to the compressor performance. Two dashed lines from node 2 to nodes 5 and 6 stand for functional dimensions of the rotor to the front cover plate and the rear cover plate, respectively. These two clearances are very important to the compressor. When the clearance is decreased, friction between the rotor and two cover plates, power loss and wear of components increase. If the clearance is increased, leakage of fluid occurs and reduces the compression performance. Therefore, all the dimen-

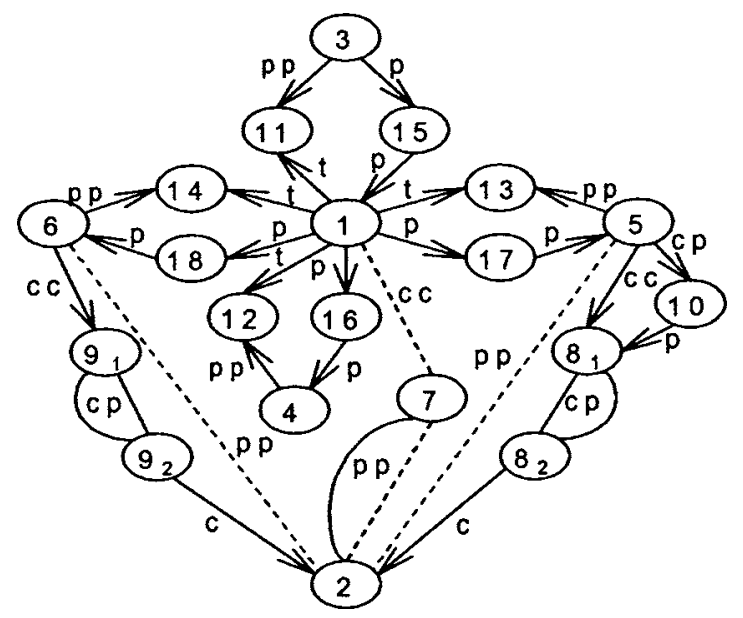

Fig. 8 Oriented functional relationship graph of rotary compressor 


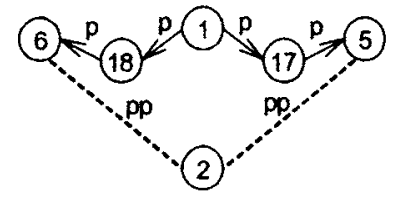

(a)

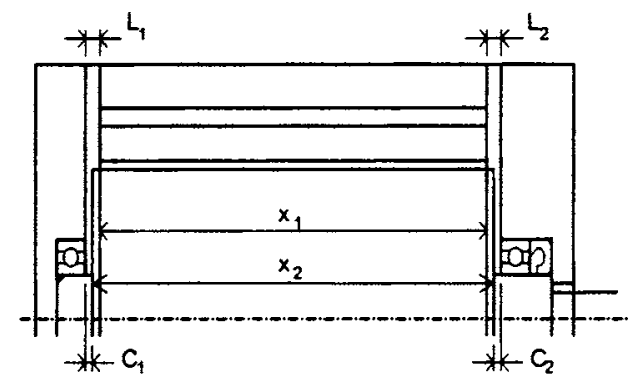

(b)

Fig. 9 First functional dimension chain

sion chains related to these two clearances need to be controlled.

The first functional dimension chain consists of six nodes as shown in Fig. 9(a). The dimension variables are shown in Fig. $9(b)$. The length of slot in the rotor, $x_{2}$, plus two specific clearances $C_{1}$ and $C_{2}$ should equal the stator length $x_{1}$ plus the thickness of two gaskets $L_{1}$ and $L_{2}$. The constraint is

$$
x_{1}+L_{1}+L_{2}=x_{2}+C_{1}+C_{2}
$$

There are four nodes in the second functional dimension chain as shown in Fig. 10(a). Nodes $9_{1}$ and $9_{2}$ stand for the outer ring and the inner ring of the bearing. The thickness of the bearing plus the wall thickness of the slot $L_{3}$ should equal the thickness of the real cover plate $x_{4}$. The locations of dimension variables are shown in Fig. 10(b). The constraint can be written as

$$
x_{3}+L_{3}+C_{1}=x_{4}+C_{1}
$$

The third functional dimension chain as shown in Fig. 11 is similar to the second functional dimension chain, except with one more oil ring, node 10 . The bearing thickness $L_{4}$, the oil ring

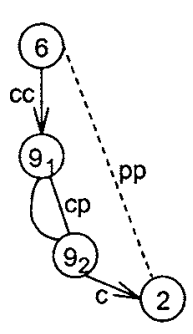

(a)

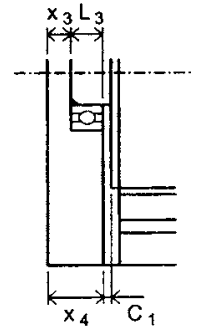

(b)
Fig. 10 Second functional dimension chain

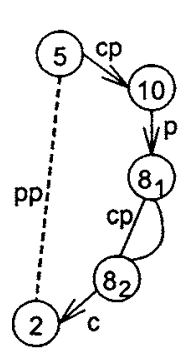

(a)

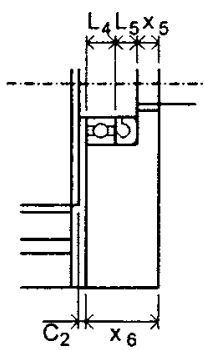

(b) thickness plus the wall thickness $x_{5}$ should be equal to the front cover plate thickness $x_{6}$ as shown in Fig. 11(b). The constraint can be written as

$$
C_{2}+L_{4}+L_{5}+x_{5}=C_{2}+x_{6}
$$

In addition, a dashed line that connects nodes 2 and 7 stands for the clearance between vanes and the rotor. The rotor and the vane form a sub-assembly, and the fourth functional dimension chain is shown in Fig. 12(a). The schematic drawing of the vane and the rotor slot is shown in Fig. 12(b), and the dimension relation is

$$
x_{7}+C_{3}+C_{4}=x_{8}
$$

Synthesis and Optimization of Tolerance. The design functions as shown in Fig. 13 can be rewritten into

$$
\begin{gathered}
\mathrm{G}_{1}=\left(x_{1}+L_{1}+L_{2}\right)-\left(x_{2}+C_{1}+C_{2}\right)+0.1 \\
\mathrm{G}_{2}=x_{3}+L_{3}-x_{4}+0.1
\end{gathered}
$$

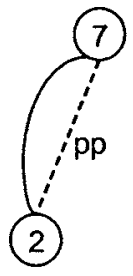

(a)

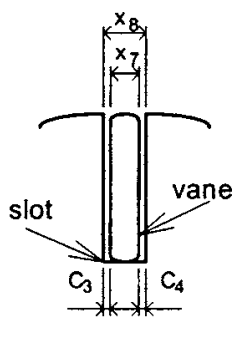

(b)
Fig. 12 Fourth functional dimension chain

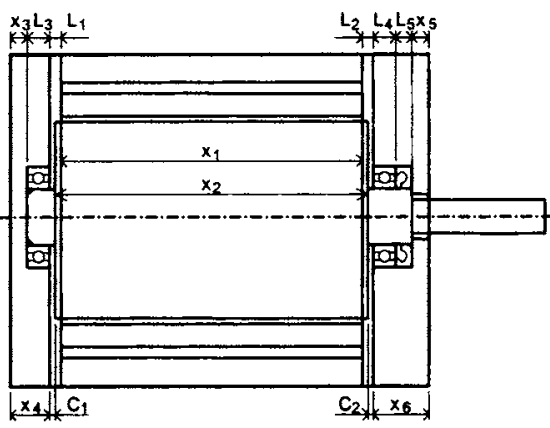

(a)

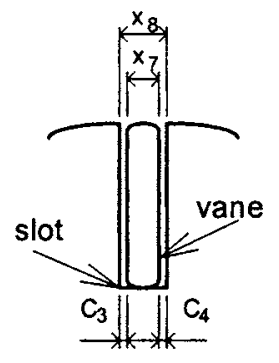

(b)
Fig. 11 Third functional dimension chain 


$$
\begin{gathered}
\mathrm{G}_{3}=x_{4}-x_{3}-L_{3}+0.1 \\
\mathrm{G}_{4}=L_{4}+L_{5}+x_{5}-x_{6}+0.1 \\
\mathrm{G}_{5}=x_{6}-L_{4}-L_{5}-x_{5}+0.1 \\
\mathrm{G}_{6}=x_{8}-\left(x_{7}+C_{3}+C_{4}\right)+0.02
\end{gathered}
$$

The design function $G_{1}$ stands for the clearance constraint between the front cover plate and the rear cover plate. The sum of clearances is within $0.1 \mathrm{~mm}$. The design functions $G_{2}$ and $G_{3}$ mean that the thickness variation between the rear cover plate and the bearing is within $0.1 \mathrm{~mm}$. The design functions $G_{4}$ and $G_{5}$ mean that the thickness variation between the front cover plate, the bearing and the oil ring is within $0.1 \mathrm{~mm}$. The purpose of the first five design functions is apparently to maintain the appropriate clearances $C_{1}$ and $C_{2}$ between two cover plates and bearings in order to prevent leakage and reduce friction. The design function $G_{6}$ stands for the required clearance between the vanes and the slots of the rotor to allow vanes to slide smoothly. The combined nominal clearance of $C_{3}$ and $C_{4}$ is $0.05 \mathrm{~mm}$ and the minimum clearance is $0.02 \mathrm{~mm}$.

The covariance matrix of dimension variables is

$$
V^{\prime}=\left[\begin{array}{cccc}
1 & \rho_{12} & \cdots & \rho_{1 n} \\
\rho_{21} & 1 & \cdots & \rho_{2 n} \\
\vdots & \vdots & \ddots & \vdots \\
\rho_{n 1} & \rho_{n 2} & \cdots & 1
\end{array}\right]
$$

According to Parkinson [9], the dimensions on the same component are correlated. It can be considered that the different dimensions at the same component may be manufactured by the same datum setup during the process. Holes for the bearings and the oil ring in the front cover plate and the rear cover plate are manufactured by the milling process. The parts are set up on the same fixture when machining for the plate thickness and the holes. The dimension variables $x_{3}$ and $x_{4}$ located at the rear cover plate are dependent, and the dimension variables $x_{5}$ and $x_{6}$ located at the front cover plate are dependent. Therefore, the values of the correlation coefficients $\rho_{34}$ and $\rho_{56}$ are not zeros. The other dimension variables are assumed independent and their correlation coefficients are zero. Equation (30) can be rewritten as

$$
V^{\prime}=\left[\begin{array}{ccccccccccc}
1.0 & 0 & 0 & 0 & 0 & 0 & 0 & 0 & 0 & 0 & 0 \\
0 & 1.0 & 0 & 0 & 0 & 0 & 0 & 0 & 0 & 0 & 0 \\
0 & 0 & 1.0 & \rho_{34} & 0 & 0 & 0 & 0 & 0 & 0 & 0 \\
0 & 0 & \rho_{43} & 1.0 & 0 & 0 & 0 & 0 & 0 & 0 & 0 \\
0 & 0 & 0 & 0 & 1.0 & \rho_{56} & 0 & 0 & 0 & 0 & 0 \\
0 & 0 & 0 & 0 & \rho_{65} & 1.0 & 0 & 0 & 0 & 0 & 0 \\
0 & 0 & 0 & 0 & 0 & 0 & 1.0 & 0 & 0 & 0 & 0 \\
0 & 0 & 0 & 0 & 0 & 0 & 0 & 1.0 & 0 & 0 & 0 \\
0 & 0 & 0 & 0 & 0 & 0 & 0 & 0 & 1.0 & 0 & 0 \\
0 & 0 & 0 & 0 & 0 & 0 & 0 & 0 & 0 & 1.0 & 0 \\
0 & 0 & 0 & 0 & 0 & 0 & 0 & 0 & 0 & 0 & 1.0
\end{array}\right]
$$

Optimal tolerance allocation can be achieved by following the process mentioned above.

\section{Results}

The combined model is evaluated for optimal tolerance allocation of an assembly with normal distributions of independent variables without the mean-shift and quality loss. The results are compared and show comparable agreement with the existing data [39] to assure the feasibility of the combined methodology.
Table 2 Statistical distribution types of dimensions

\begin{tabular}{ccc}
\hline \hline Symbol & Name & Distribution type \\
\hline$x_{1}$ & Stator length & Lognormal \\
$x_{2}$ & Rotor center length & Lognormal \\
$x_{3}$ & Rear cover plate sink thickness & Lognormal \\
$x_{4}$ & Rear cover plate thickness & Lognormal \\
$x_{5}$ & Front cover plate sink thickness & Lognormal \\
$x_{6}$ & Front cover plate thickness & Lognormal \\
$x_{7}$ & Vane thickness & Lognormal \\
$x_{8}$ & Slot width & Lognormal \\
$L_{1}$ & Gasket thickness & Normal \\
$L_{2}$ & Gasket thickness & Normal \\
$L_{3}$ & Real cover plate bearing thickness & Normal \\
$L_{4}$ & Front cover plate bearing thickness & Normal \\
$L_{5}$ & Oil ring thickness & Normal \\
$C_{1}$ & Rear cover plate clearance & Fixed target value \\
$C_{2}$ & Front cover plate clearance & Fixed target value \\
$C_{3}$ & Clearance between vane and slot & Fixed target value \\
$C_{4}$ & Clearance between vane and slot & Fixed target value \\
\hline \hline
\end{tabular}

The statistical distributions of components depend on manufacturing processes. The oil ring, bearings, and gaskets are standard parts made in mass production, and the dimensions are assumed to be normal distributions. The other components are manufactured in batch production, and the dimensions are assumed to be lognormal distributions as shown in Table 2 .

Only the lognormal distributions of dimension variables $x_{1}$ to $x_{8}$ will be optimized, and these tolerances must be specified in design drawings. Dimension variables $L_{1}$ to $L_{5}$ are from standard parts made in mass production and will not be optimized. Normal distributions of these dimensions affect the reliability index in the reliability model. Hence, the standard deviations of these dimensions must be controlled to have good quality products.

Since costs of some compressor components are not available, the process of this study is based on the cost tolerance model proposed by Bjørke [6]. The numerical function in the reciprocal power type is used for the curve fitting, and the coefficients of the curve are listed in Table 3 . The cost tolerance coefficients of all functional dimensions of components are listed in Table 4. Because $L_{1}$ to $L_{5}$ are the dimensions of the standard parts and $C_{1}$ to $C_{4}$ are specified clearances, they are not included in the optimization model. If the required reliability is $75 \%$ and the required reliability index $\beta^{*}$ is equal to 0.67449 , customer's semitolerances of all dimension variables are listed in Table 5.

Based on the manufacturing cost, the replacement costs of the stator $A_{1}$, the rotor $A_{2}$, the rear cover plate $A_{3}$, the front cover plate $A_{4}$, and the set of vanes $A_{5}$ are 9.0, 5.0, 1.0, 1.5, and 0.3, respectively [6]. Quality loss coefficients of the functional dimensions obtained are listed in Table 6 . Because the replacement cost of the rotor is high and the customer's semi-tolerances of $x_{2}$ and $x_{8}$ are smaller than other dimensions, the quality loss coefficients of $x_{2}$ and $x_{8}$ are much larger than other dimension variables. It can be expected that the dimension variables with the required tightest tolerances are $x_{2}$ and $x_{8}$ in tolerance optimization.

Table 3 Coefficients of curve fitting with $C_{m}=c_{0} t^{-c_{1}}$ for Bjørke's cost-tolerance models

\begin{tabular}{lcrc}
\hline \hline No. & Process type & \multicolumn{1}{c}{$c_{0}$} & $c_{1}$ \\
\hline 1 & External cylindrical surface (rotating) & 1.192 & 0.591 \\
2 & Internal cylindrical surface (rotating) & 7.602 & 0.814 \\
3 & Face surrounding bore, to a datum (rotating) & 18.064 & 1.115 \\
4 & Internal cylindrical surfaces (non-rotating) & 12.435 & 0.566 \\
5 & Face surrounding a bore, to a datum (non-rotating) & 18.622 & 0.564 \\
6 & Plane surface & 13.457 & 0.764 \\
\hline \hline
\end{tabular}


Table 4 Nominal dimensions and coefficients of cost tolerance function $C_{m}=c_{0} t^{c_{1}}$

\begin{tabular}{cccc}
\hline \hline & & \multicolumn{2}{c}{ Coefficients of cost tolerance function } \\
\cline { 3 - 4 } Symbol & $\begin{array}{c}\text { Nominal dimension } \\
(\mathrm{mm})\end{array}$ & $c_{0}$ & $c_{1}$ \\
\hline$x_{1}$ & 152.0 & 18.622 & 0.564 \\
$x_{2}$ & 154.8 & 18.064 & 1.115 \\
$x_{3}$ & 5.0 & 12.435 & 0.566 \\
$x_{4}$ & 20.0 & 13.457 & 0.764 \\
$x_{5}$ & 5.0 & 12.435 & 0.566 \\
$x_{6}$ & 30.0 & 13.457 & 0.764 \\
$x_{7}$ & 4.95 & 13.457 & 0.764 \\
$x_{8}$ & 5.0 & 13.457 & 0.764 \\
$L_{1}$ & 1.5 & & \\
$L_{2}$ & 1.5 & & \\
$L_{3}$ & 15.0 & & \\
$L_{4}$ & 15.0 & & \\
$L_{5}$ & 10.0 & & \\
$\mathrm{C}_{1}$ & 0.1 & & \\
$\mathrm{C}_{2}$ & 0.1 & & \\
$C_{3}+C_{4}$ & 0.05 & & \\
\hline \hline
\end{tabular}

Distribution of Vane Thickness. Two sets of 10 vanes, a total of 20 vanes, are measured by a digital micrometer to obtain the mean-shift value of the thickness. The thickness of each vane is measured seven times. The largest and the smallest values are obsolete, and the other five values are used to calculate the mean value to reduce the measured error. The mean values of the measured vane thickness are listed in Table 7 .

Table 5 Customer's semi-tolerance of dimension variables

\begin{tabular}{ccc}
\hline \hline Symbol & $\begin{array}{c}\text { Left customer's } \\
\text { semi-tolerance } \Delta_{i L}(\mathrm{~mm})\end{array}$ & $\begin{array}{c}\text { Right customer's } \\
\text { semi-tolerance } \Delta_{i R}(\mathrm{~mm})\end{array}$ \\
\hline$x_{1}$ & 0.3341 & 0.3347 \\
$x_{2}$ & 0.1303 & 0.1304 \\
$x_{3}$ & 0.2808 & 0.2945 \\
$x_{4}$ & 0.2103 & 0.2121 \\
$x_{5}$ & 0.2801 & 0.2938 \\
$x_{6}$ & 0.2100 & 0.2112 \\
$x_{7}$ & 0.0509 & 0.0513 \\
$x_{8}$ & 0.0507 & 0.0511 \\
\hline \hline
\end{tabular}

Table 6 Quality loss coefficients of functional dimensions

\begin{tabular}{ccc}
\hline \hline Symbol & $\begin{array}{c}\text { Left quality } \\
\text { loss coefficient } k_{i L}\end{array}$ & $\begin{array}{c}\text { Right quality loss } \\
\text { coefficient } k_{i R}\end{array}$ \\
\hline$x_{1}$ & 80.6491 & 80.3548 \\
$x_{2}$ & 294.4430 & 294.0312 \\
$x_{3}$ & 12.6799 & 11.5238 \\
$x_{4}$ & 22.6086 & 22.2152 \\
$x_{5}$ & 19.1211 & 17.3823 \\
$x_{6}$ & 34.0203 & 33.6257 \\
$x_{7}$ & 115.9523 & 113.9805 \\
$x_{8}$ & 3500.6748 & 3441.9239 \\
\hline
\end{tabular}

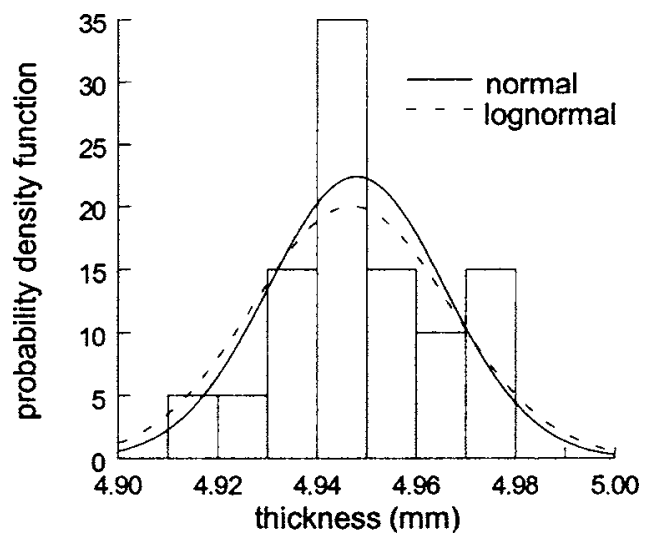

Fig. 14 Probability density functions of normal and lognormal distributions for vane thickness

The mean value and the standard deviation of the normal distribution for the vane thickness are 4.948 and 0.0178 , respectively. The middle value and the coefficient of variation of the lognormal distribution are 4.947 and 0.0403 , respectively. The curves of the probability density function of the normal distribution and the lognormal distribution and the practical distribution histogram are shown in Fig. 14.

The chi-square test is used to verify the fitting goodness of the normal distribution and the lognormal distribution. The results are shown in Table 8 . The symbol $n_{i}$ is the observed frequency for each interval and $e_{i}$ is the theoretical frequency. From the test results, fitting of the lognormal distribution is better than that of the normal distribution for the vane thickness.

Since only the dimension variables of the lognormal distributions are used for tolerance optimization in this study, three parameters that are needed for optimization are the mean-shift value, the correlation coefficient of the lognormal distribution, and the standard deviation of dimension variables of the normal distribution. First, it is assumed that the vanes, the front cover plate, and the real cover plate are to be processed by the same machine. Therefore, based on the mean-shift value of the vane thickness $x_{7}$, the mean-shift values of the rear cover plate $x_{4}$ and the front cover plate $x_{6}$ are assumed to be $0.003 \mathrm{~mm}$. It is assumed that other dimensions do not have the mean-shift. Second, the correlation coefficients of two parts depend on the machining process and the fixture datum setup. In this case, the dimensions of $x_{3}$ and $x_{4}$, and $x_{5}$ and $x_{6}$ are assumed to be independent. Third, the statistical data of the mass production parts are not available. So, it is assumed that standard deviations of these standard parts are $0.01 \mathrm{~mm}$. Tolerances of these standard parts are $0.06 \mathrm{~mm}$ obtained from the concept of six-sigma, $\pm 3 \sigma$. If all of the correlation coefficients are assumed to be zero, the result of tolerance optimization is listed in Table 9. The dimensions with specified tolerances for practical engineering drawings are listed in Table 10. The upper and lower tolerances of functional dimensions are shown in Fig. 15.

The correlation coefficients $\rho_{34}$ of $x_{3}$ and $x_{4}$ and $\rho_{56}$ of $x_{5}$ and $x_{6}$ can be set as variables based on optimal tolerances obtained to perform the parameter analysis. Since the machining processes are similar, these two correlation coefficients are set as the same vari-

Table 7 Mean values of measured vane thickness

\begin{tabular}{cccccccc}
\hline \hline No & Thickness $(\mathrm{mm})$ & No & Thickness $(\mathrm{mm})$ & No & Thickness $(\mathrm{mm})$ & No & Thickness (mm) \\
\hline 1 & 4.917 & 6 & 4.949 & 11 & 4.976 & 16 & 4.943 \\
2 & 4.950 & 7 & 4.958 & 12 & 4.945 & 17 & 4.945 \\
3 & 4.941 & 8 & 4.946 & 13 & 4.939 & 18 & 4.969 \\
4 & 4.935 & 9 & 4.951 & 14 & 4.925 & 19 & 4.974 \\
5 & 4.930 & 10 & 4.949 & 15 & 4.965 & 20 & 4.977 \\
\hline \hline
\end{tabular}


Table 8 Normal distribution and lognormal distribution verified by chi-square test

\begin{tabular}{|c|c|c|c|c|c|}
\hline \multirow{2}{*}{$\begin{array}{l}\text { Interval } \\
(\mathrm{mm})\end{array}$} & \multirow{2}{*}{$\begin{array}{c}\text { Real } \\
\text { number } n_{i}\end{array}$} & \multicolumn{2}{|c|}{$\begin{array}{l}\text { Theoretical number } \\
\qquad e_{i}\end{array}$} & \multicolumn{2}{|c|}{$\begin{array}{c}\text { chi-square value } \\
\quad\left(n_{i}-e_{i}\right)^{2} / e_{i}\end{array}$} \\
\hline & & Normal & Lognormal & Normal & Lognormal \\
\hline$<4.920$ & 1 & 1.16 & 1.74 & 0.022 & 0.315 \\
\hline $4.920-4.930$ & 1 & 1.97 & 2.19 & 0.478 & 0.647 \\
\hline $4.930-4.940$ & 3 & 3.40 & 3.32 & 0.047 & 0.031 \\
\hline $4.940-4.950$ & 7 & 4.36 & 4.72 & 1.599 & 1.101 \\
\hline $4.950-4.960$ & 3 & 4.11 & 2.87 & 0.300 & 0.006 \\
\hline $4.960-4.970$ & 2 & 2.84 & 2.66 & 0.248 & 0.164 \\
\hline$>4.970$ & 3 & 2.16 & 2.50 & 0.327 & 0.100 \\
\hline Sum & 20 & 20.0 & 20.0 & 3.021 & 2.364 \\
\hline
\end{tabular}

able $\rho$. The relation between the correlation coefficient $\rho$ and the cost is shown in Fig. 16. The second parameter analysis performed is to set the standard deviations of the mass production parts as the variables and analyze the relation between the quality of standard parts and the cost. The result is shown in Fig. 17. Increasing the weighting ratio $W_{q} / W_{m}$ increases the relative im-

Table 9 Results of normal distribution with standard deviation of $0.01 \mathrm{~mm}$ and zero correlation coefficients

\begin{tabular}{ccccc}
\hline \hline Variable & $\begin{array}{c}\text { Optimum standard } \\
\text { deviation }(\mathrm{mm})\end{array}$ & $\begin{array}{c}\text { Lower limit of } \\
\text { tolerance }(\mathrm{mm})\end{array}$ & $\begin{array}{c}\text { Upper limit of } \\
\text { tolerance }(\mathrm{mm})\end{array}$ & $\begin{array}{c}\text { Total } \\
\text { tolerance } \\
(\mathrm{mm})\end{array}$ \\
\hline$x_{1}$ & 0.03788 & 0.0921 & 0.0921 & 0.1842 \\
$x_{2}$ & 0.01461 & 0.0355 & 0.0355 & 0.0710 \\
$x_{3}$ & 0.03168 & 0.0766 & 0.0776 & 0.1542 \\
$x_{4}$ & 0.02510 & 0.0610 & 0.0611 & 0.1221 \\
$x_{5}$ & 0.03078 & 0.0744 & 0.0753 & 0.1497 \\
$x_{6}$ & 0.02423 & 0.0589 & 0.0590 & 0.1179 \\
$x_{7}$ & 0.00753 & 0.0183 & 0.0183 & 0.0366 \\
$x_{8}$ & 0.00640 & 0.0155 & 0.0156 & 0.0311 \\
\multicolumn{5}{c}{ Optimum machining cost $C_{m}$ is 5.1128 } \\
\multicolumn{5}{c}{ The result of Monte Carlo simulation is $93.71 \%$ by 10,000 samples }
\end{tabular}

Table 10 Functional dimensions with optimal tolerances specified

\begin{tabular}{ccc}
\hline \hline Variable & Dimension description & Dimension (mm) \\
\hline$x_{1}$ & Stator length & $152 \pm 0.092$ \\
$x_{2}$ & Rotor slot length & $154.8 \pm 0.036$ \\
$x_{3}$ & Rear cover plate wall thickness with cut off & $5+0.077$ \\
& & -0.078 \\
$x_{4}$ & Rear cover plate thickness & $20 \pm 0.061$ \\
$x_{5}$ & Front cover plate thickness with cut off & $5+0.074$ \\
& & -0.075 \\
$x_{6}$ & Front cover plate thickness & $30 \pm 0.059$ \\
$x_{7}$ & Vane thickness & $4.95 \pm 0.018$ \\
$x_{8}$ & Rotor slot width & $5 \pm 0.016$ \\
\hline \hline
\end{tabular}

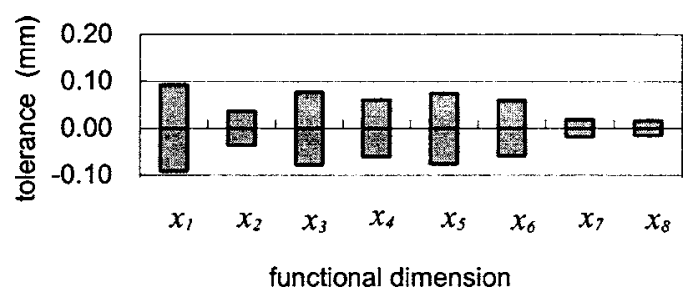

Fig. 15 Upper and lower tolerances of function dimensions

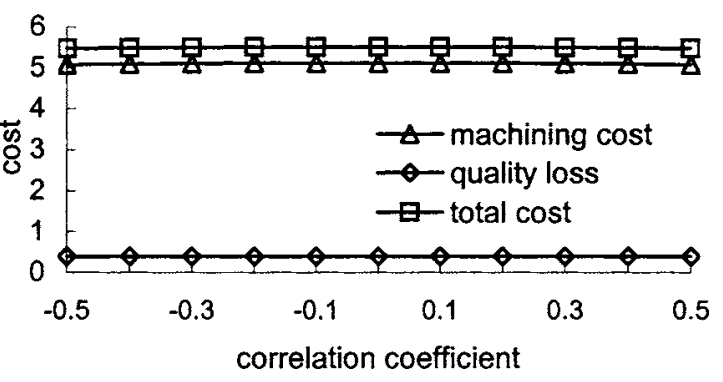

Fig. 16 Relation between correlation coefficient of cover plate thickness and cost

portance of quality loss in the combined objective function. The effect of weighting ratio on the cost is studied, and the results are shown in Fig. 18.

\section{Discussion}

The required reliability, the minimum machining cost and quality loss are considered simultaneously for tolerance allocation design to improve existing models. Consideration of the required reliability, the minimum machining cost and quality loss simultaneously is a multi-attribute decision-making problem. In general, the multi-attribute utility analysis is a good way to obtain the optimum results in many applications. Since the manufacturing costs of compressor components are obtained from machining factories in this study, and the replacement cost $A_{h}$ is converted into the equivalent cost as the same unit as Bjørke's cost-tolerance model, the gradients of the distribution curves can be obtained. The advantage of single-objective optimization is that the efficiency is higher and calculation time is less than the multiattribute utility analysis. Therefore, the Beta algorithm process and the non-linear programming are used in this study. Hohenbichiler and Rackwitz's Beta algorithm is used to calculate the reliability index [35] because the efficiency of the transformation is higher than those of other transformation methods when the distribution model is non-normal and asymmetric.

Table 5 shows that right customer's semi-tolerance $\Delta_{i R}$ is larger than left customer's semi-tolerance $\Delta_{i L}$. Therefore, the left quality loss coefficient $k_{i L}$ is larger than the right quality loss coefficient

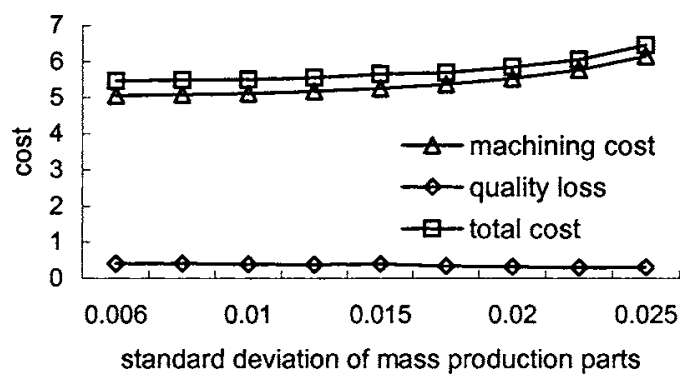

Fig. 17 Relation between standard deviations of mass production parts and cost

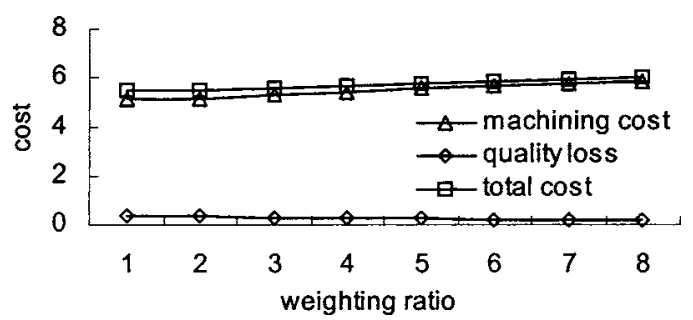

Fig. 18 Relation between weighting ratio and cost 
$K_{i R}$ as shown in Table 6. The quality loss coefficients of $x_{2}$ and $x_{8}$ are much higher than those of other dimensions because the stator and the rotor are the most expensive components in the rotary compressor.

The chi-square value 2.364 of the lognormal distribution is smaller than the value 3.021 of the normal distribution as shown in Table 8. It demonstrates that the lognormal distribution has better fitting than the normal distribution for the vane thickness when mean-shift and asymmetry exist. The mean value of vanes as shown in Table 7 is $4.947 \mathrm{~mm}$ and the nominal dimension as shown in Table 4 is $4.95 \mathrm{~mm}$. The mean-shift value of the lognormal distribution due to machining is $0.003 \mathrm{~mm}$, which is used for tolerance optimization in this study.

Design functions control tolerances of the vane thickness and the slot width, and the slot length affects clearances of the front cover plate and the rear cover plate. The optimization generates tighter tolerances for the slot length $x_{2}$, the vane thickness $x_{7}$ and the slot width $x_{8}$, which are critical function dimensions for manufacturing, than other functional dimensions as shown in Fig. 15. The unit value of $0.01 \mathrm{~mm}$ is used in Table 10 in order to match the precision of the regular machining process.

When correlation coefficients $\rho_{x_{3} x_{4}}$ of $x_{3}$ and $x_{4}$ and $\rho_{x_{5} x_{6}}$ of $x_{5}$ and $x_{6}$ vary, the cost variation is insignificant as shown in Fig. 16. One reason is that dimension variables $x_{3}$ and $x_{4}$, and $x_{5}$ and $x_{6}$ have larger tolerances than others. The variation of correlation coefficients does not affect the total cost significantly. When the standard deviation of standard parts increases, the total cost increases as shown in Fig. 17. It means that other components of the assembly made by batch production need to have high manufacturing accuracy in order to compensate for poor quality mass production parts.

The quality loss in the total cost function is small compared with the total cost as shown in Figs. 16 and 17. The effect of the weighting ratio $W_{q} / W_{m}$ varied from 1 to 8 on the cost is investigated. The results as shown in Fig. 18 indicate that the cost of quality loss is reduced when the weighting ratio is increased. The total cost is increased because tight tolerance allocation increases the machining cost. However, the situation can be changed if the part is made of high-cost material and the replacement cost of a component is much greater than the machining cost.

\section{Conclusion}

An optimization model has been established with consideration of the required functional reliability, the minimum machining cost and quality loss in this study. The procedure to obtain optimal customer's semi-tolerance with the combined model is revealed. The reliability index model is capable of handling the normal distribution and the non-normal distribution. Tolerance allocation of the compressor is optimized. The data of the vane thickness is better fitted by the lognormal distribution than the normal distribution, and it is verified by chi-square test.

The oriented relationship functional graph has been used to analyze the dimension connection of the components in the sliding vane rotary compressor. Through the graph analysis, the functional dimension chains are sorted out and then the design functions for optimization are generated. Optimal tolerance allocation of functional dimensions in the rotary compressor is obtained by the non-linear programming with the efficient beta algorithm [35]. According to the optimization results, tolerances of the rotor slot length, the rotor slot width, and the vane thickness are tighter than those of other functional dimensions. Furthermore, the effect of correlation coefficients has been analyzed. The effects of the correlation coefficients of the bottom cover plate and the top cover plate, and the front cover plate and the rear cover plate on the cost are insignificant. When the standard deviation of standard parts increases, the total cost increases because other components of the assembly made by batch production need to have high manufacturing accuracy in order to compensate for poor quality mass production parts. The cost of quality loss is reduced when the weight- ing ratio is increased. The total cost is increased because tight tolerance allocation increases the machining cost.

\section{Acknowledgments}

The authors would like to express their sincere thanks to the National Science Council of the Republic of China for Grant No. NSC 93-2218-E-002-063 for funding this investigation and to Grant D. Huang for comments and revisions made on this manuscript.

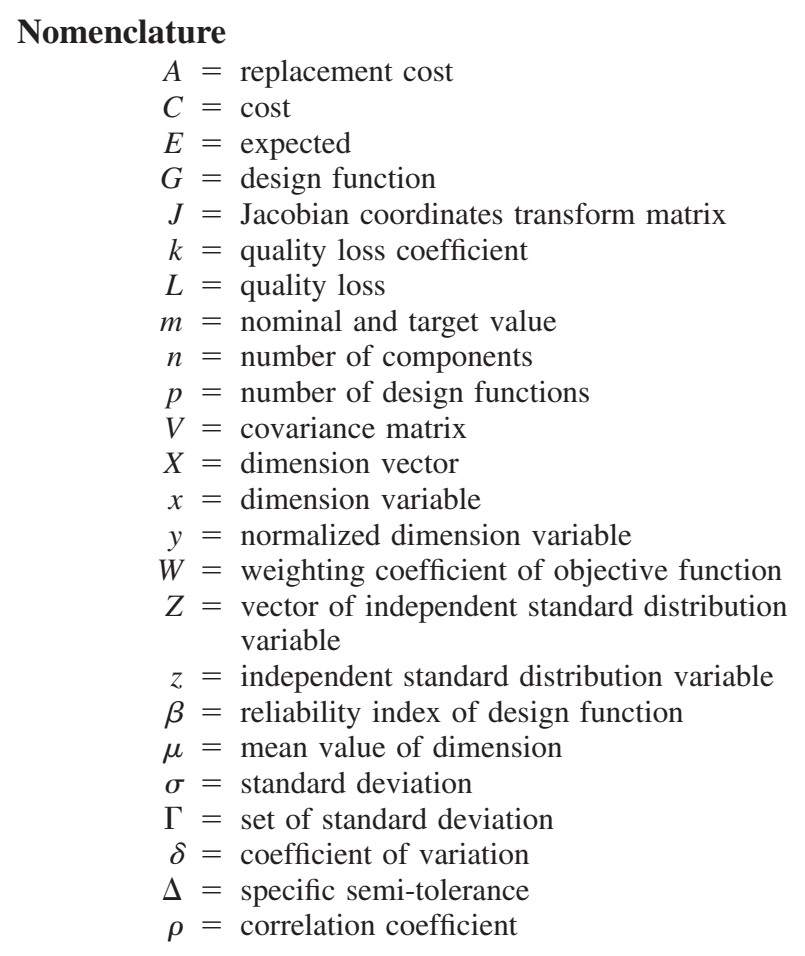

Subscripts

$$
\begin{aligned}
0 & =\text { initial condition } \\
1,2, \ldots, n & =\text { dimension } \\
\text { asm } & =\text { assembly } \\
h & =h \text { th component } \\
i & =i \text { th dimension } \\
j & =j \text { th dimension } \\
i j & =\text { relation between } i \text { th and } j \text { th dimension } \\
k & =k \text { th design function } \\
L & =\text { left side of mean value } \\
m & =\text { machining } \\
\min & =\text { minimum } \\
q & =\text { quality loss } \\
R & =\text { right side of mean value } \\
t & =\text { total } \\
x & =\text { dimension variables } \\
z & =\text { normalized dimension variable }
\end{aligned}
$$

$$
\begin{aligned}
\text { Superscripts } & \\
*^{*} & =\text { required } \\
- & =\text { mean } \\
\mathrm{t} & =\text { transport }
\end{aligned}
$$

\section{Appendix}

The beta algorithm that was developed by Hohenbichler and Rackwitz [35] is an iteration method to calculate the reliability index. The steps for the normal distribution or the lognormal distribution are listed as follows [40]:

a. Select a starting point in the variable space, the vector $X_{0}$. 
b. Using Rosenblatt transformation to transfer $X_{0}$ into an independent normalized vector $Z_{0}$.

c. Determine the Jacobian matrix

$$
J=\frac{\partial\left(z_{1}, z_{2}, \ldots, z_{n}\right)}{\partial\left(x_{1}, x_{2}, \ldots, x_{n}\right)}=\left[\begin{array}{cccc}
\frac{\partial z_{1}}{\partial x_{1}} & \frac{\partial z_{1}}{\partial x_{2}} & \cdots & \frac{\partial z_{1}}{\partial x_{n}} \\
\frac{\partial z_{2}}{\partial x_{1}} & \frac{\partial z_{2}}{\partial x_{2}} & \cdots & \frac{\partial z_{2}}{\partial x_{n}} \\
\vdots & \vdots & \ddots & \vdots \\
\frac{\partial z_{n}}{\partial x_{1}} & \frac{\partial z_{n}}{\partial x_{2}} & \cdots & \frac{\partial z_{n}}{\partial x_{n}}
\end{array}\right]
$$

d. Obtain the value of the design function $G$ and its gradient $\nabla G$

$$
\begin{gathered}
G_{z}\left(Z_{0}\right)=G_{X}\left(X_{0}\right) \\
\nabla G_{Z}=\left(J^{-1}\right)^{t} \nabla G_{X}
\end{gathered}
$$

e. The new point can be obtained from the gradient of the vector

$$
Z^{*}=\frac{1}{\nabla G_{Z}^{t} \nabla G_{Z}}\left[\nabla G_{Z}^{t} Z_{0}-G_{Z}\left(Z_{0}\right)\right] \nabla G_{Z}
$$

f. The new iteration point in $X$ space can be approached by the first-order linear approximation

$$
X^{*} \cong X_{0}+J^{-1}\left(Z^{*}-Z_{0}\right)
$$

g. Obtain the reliability index for this iteration

$$
\beta=\left(Z^{*} Z^{*}\right)^{1 / 2}
$$

h. Input $X^{*}$ into the iteration process again until the convergence is reached.

\section{References}

[1] Huang, Y. M., 1995, "Analysis of the Roto-Cooler Air-Conditioning System," Refrigeration, 18, pp. 367-372.

[2] Huang, Y. M., and Huang, C.-T., 2002, "Disassembly Matrix for Disassembly Processes of Products," Int. J. Prod. Res., 40, pp. 255-273.

[3] Huang, Y. M., and Chang, C. H., 1997, "The Stress and Deflection Analysis for the Radial Blade of the RCAC System," Refrigeration, 20, pp. 55-62.

[4] Huang, Y. M., and Liaw, Y.-S., 2001, "The Impact of Sliding Blades in a Rotary Compressor," ASME J. Mech. Des., 123, pp. 583-589.

[5] Huang, Y. M., and Yang, S. A., 2002, "Evaluation of Performance for a Rotary Vane Compressor," Seventh NRC/ASME Symposium on Valve and Pump Testing, NUREG/CP-0152, 4, pp. 3A1-3A16.

[6] Bjørke, Ø., 1989, Computer Aided Tolerancing, 2nd Edition, ASME Press.

[7] Juster, N. P., Dew, P. M., and Pennington, A., 1992, "Automating Linear Tolerance Analysis Across Assemblies," ASME J. Mech. Des., 114, pp. 174179.

[8] Feng, S. C., and Yang, Y., 1995, "A Dimension and Tolerance Data Model for Concurrent Design and Systems Integration,” J. Manuf. Syst., 14, pp. 406426.

[9] Parkinson, D. B., 2000, "The Application of a Robust Design Method to Tolerancing," ASME J. Mech. Des., 122, pp. 149-154.

[10] Zhou, Z., Huang, W., and Zhang, L., 2001, "Sequential Algorithm Based on Number Theoretic Method for Statistical Tolerance Analysis and Synthesis," ASME J. Manuf. Sci. Eng., 123, pp. 490-493.

[11] Jordaan, J. P., and Ungerer, C. P., 2002, "Optimization of Design Tolerances Through Response Surface Approximations,” ASME J. Manuf. Sci. Eng., 124, pp. 762-767.

[12] Di Stefano, P., 2003, "Tolerance Analysis and Synthesis Using the Mean Shift Model,” Proc. Inst. Mech. Eng., Part C: J. Mech. Eng. Sci., 217, pp. 149-159.

[13] Hong, Y. S., and Chang, T. C., 2003, "Tolerancing Algebra: A Building Block for Handling Tolerance Interactions in Design and Manufacturing," Int. J. Prod. Res., 41, pp. 47-63.

[14] Marin, R. A., and Ferreira, P. M., 2003, "Analysis of the Influence of Fixture Locator Errors on the Compliance of Work Part Features to Geometric Tolerance Specifications," ASME J. Manuf. Sci. Eng., 125, pp. 609-616.

[15] Manarvi, I. A., and Juster, N. P., 2004, "Framework of an Integrated Tolerance Synthesis Model and Using FE Simulation as a Virtual Tool for Tolerance Allocation in Assembly Design," J. Mater. Process. Technol., 150, pp. 182 193.

[16] Hu, J., Xiong, G., and Wu, Z., 2004, “A Variational Geometric Constraints Network for a Tolerance Type Specification,” Int. J. Adv. Manuf. Technol., 24, pp. 214-222.

[17] Wu, W., and Rao, S. S., 2004, "Interval Approach for the Modeling of Tolerances and Clearances in Mechanism Analysis," ASME J. Mech. Des., 126, pp. 581-592.

[18] Mujezinovi'c, A., Davidson, J. K., and Shah, J. J., 2004, “A New Mathematical Model for Geometric Tolerances as Applied to Polygonal Faces," ASME J. Mech. Des., 126, pp. 504-518.

[19] Dong, Z., Hu, W., and Xue, D., 1994, "New Production Cost-Tolerance Models for Tolerance Synthesis,” ASME J. Eng. Ind., 116, pp. 199-206.

[20] Shiu, W., Apley, D. W., Geglarek, D., and Shi, J., 2003, "Tolerance Allocation for Compliant Beam Structure Assemblies," IIE Trans., 35, pp. 329-342.

[21] Xue, J., and Ji, P., 2004, "Process Tolerance Allocation in Angular Tolerance Charting," Int. J. Prod. Res., 42, pp. 3929-3945.

[22] Mahadevan, S., and Ni, K., 2003, "Damage Tolerance Reliability Analysis of Automotive Spot-Welded Joints," Reliab. Eng. Syst. Saf., 81, pp. 9-21.

[23] Söderberg, R., 1994, "Tolerance Allocation Considering Customer and Manufacturer Objectives," Adv. Design Automation, ASME DE, 65-2, pp. 149-157.

[24] Jeang, A., 1994, "Tolerance Design: Choosing Optimal Tolerance Specifications in The Design of Machined Parts," Qual. Reliab. Eng. Int, 10, pp. 27-35.

[25] Krishnaswami, M., and Mayne, R. W., 1994, "Optimizing Tolerance Allocation Based on Manufacturing Cost and Quality Loss," Adv. Design Automation, ASME DE, 69-1, pp. 211-217.

[26] Cheng, B. W., and Maghsoodloo, S., 1995, "Optimization of Mechanical Assembly Tolerances by Incorporating Taguchi's Quality Loss Function," J. Manuf. Syst., 14, pp. 264-276.

[27] Ye, B., and Salustri, F. A., 2003, "Simultaneous Tolerance Synthesis for Manufacturing and Quality,” Res. Eng. Des., 14, pp. 98-106.

[28] Li, M. H. C., 2004, "Optimal Target Selection for Unbalanced Tolerance Design," Int. J. Adv. Manuf. Technol., 23, pp. 743-749.

[29] Lee, J., and Johnson, G. E., 1993, "Optimal Tolerance Allotment Using a Genetic Algorithm and Truncated Monte Carlo Simulation," Comput.-Aided Des., 25, pp. 601-611.

[30] Skowronski, V. J., and Turner, J. U., 1997, "Using Monte-Carlo Variance Reduction in Statistical Tolerance Synthesis," Comput.-Aided Des., 29, pp. 63-69.

[31] Vasseur, H., Kurfess, T. R., and Cagan, J., 1997, "Use of a Quality Loss Function to Select Statistical Tolerances," ASME J. Manuf. Sci. Eng., 119, pp. 410-416.

[32] Choi, H. G., Park, M. H., and Salisbury, E., 2000, "Optimal Tolerance Allocation With Loss Functions,” ASME J. Manuf. Sci. Eng., 122, pp. 529-535.

[33] Greenwood, W. H., and Chase, K. W., 1987, "A New Tolerance Analysis Method for Designers and Manufacturers," ASME J. Eng. Ind., 109, pp. 112 116.

[34] Rao, S. S., 1992, Reliability-Based Design, McGraw-Hill, New York.

[35] Hohenbichler, M., and Rackwitz, R., 1981, "Non-Normal Dependent Vectors in Structural Safety," J. Eng. Mech. Div., Am. Soc. Civ. Eng., 107, pp. 1227 1238 .

[36] Taguchi, G., and Wu, Y., 1985, Introduction to Off-Line Quality Control, Central Japan Quality Control Association.

[37] Zhang, G., and Porchet, M., 1993, "Some New Developments in Tolerance Design in CAD," Advances in Design Automation, ASME DE, 65-2, pp. 175-185.

[38] Honda, I., Ohba, H., Soejima, M., and Nakashima, Y., 1990, "Analysis of a Gap Flow on the Side Plate for Rotary Vane Compressor," Nippon Kikai Gakkai Ronbunshu, JSME, 56, pp. 1607-1610.

[39] Hohenbichler, M., and Rackwitz, R., 1981, "Non-Normal Dependent Vectors in Structural Safety," J. Eng. Mech. Div., Am. Soc. Civ. Eng., 107, pp. 1227 1238.

[40] Ang, A. H. S., and Tang, W. H., 1984, Probability Concepts in Engineering Planning and Design. Vol. II-Decision, Risk, and Reliability, John Wiley, New York. 